\title{
Factores de riesgo psicosocial y estrés percibido en trabajadores de una empresa eléctrica en Chile
}

\author{
Psychosocial Risk factors at work and perceived stress in workers of a electric \\ energy company in Chile
}

\author{
Marjory Güilgüiruca Retamal', Karina Meza Godoy', Rodolfo Góngora Cabrera², Cristóbal Moya Cañas' \\ 1. Universidad de Chile \\ 2. Universidad Católica de Chile
}

Recibido: 18-11-14

Aceptado: 09-02-15

\section{Correspondencia}

Marjory Güilgüiruca R: mguilgui@gmail.com

Av. C. Ignacio Carrera Pinto 1045, Ñuñoa, Santiago- Chile

Facultad de Ciencias Sociales.

Universidad de Chile

\section{Resumen}

Objetivos: El objetivo de esta investigación fue determinar la correlación entre riesgos psicosociales laborales y estrés percibido en trabajadores de una empresa del rubro eléctrico en Chile

Material y Métodos: Se diseñó un estudio descriptivo, de alcance asociativo y de corte transversal, en el cual participaron 292 trabajadores. Se aplicó el instrumento SUSESO ISTAS-21, versión corta de la Superintendencia de Seguridad Social (SUSESO) del Gobierno de Chile y la Escala de Estrés Percibido validada para población chilena. El análisis estadístico se realizó con el programa SPSS versión 17.0.

Resultados: Los resultados indican que todos los factores de riesgos psicosociales estudiados presentan asociaciones estadísticamente significativas con estrés percibido. Destacan las relaciones de apoyo social y exigencias psicológicas con estrés percibido, así como la relación entre género y doble presencia, antigüedad laboral y trabajo activo, sistema de turno y compensaciones.

Conclusiones: Los trabajadores evaluados manifiestan que en la medida que aumenta la percepción de riesgo de los factores psicosociales del trabajo, también aumentan sus niveles de estrés percibido con aspectos del contenido y organización del trabajo.

Med Segur Trab (Internet) 2015; 61 (238) 57-67

Palabras Clave: Factores de riesgo psicosocial, estrés percibido, trabajo.

Abstract

Objectives: The purpose of this investigation was to determine the correlation between the psychosocials risks of work and perceived stress in workers of an electric energy company in Chile.

Methods: The study was descriptive, associative and transverse, in which 292 workers participated. The short version of the Government of Chile Questionnaire SUSESO ISTAS-21 (Superintendencia de Seguridad Social) was used. The perceived stress scale was validated for the Chilean population. To Statistical analysis was used the SPSS 17.0. 
Results: The results indicate that all psychosocial risk factors of the job are associated with perceived stress in the form of significant statistics. The associations more important are between social support and psychological demands with perceived stress, between gender and double presence, years with the company and active job, shift schedules and compensations.

Conclusions: The workers evaluated show the perceived stress increase, when the psychosocials risks of work also increase. Therefore, the perceived stress increase when these workers perceive problems with contents and organization of job.

Med Segur Trab (Internet) 2015; 61 (238) 57-67

Key words: Psychosocial risk factors at the work; perceived stress, job. 


\section{INTRODUCCIÓN}

El fenómeno del estrés laboral, ha sido reconocido como uno de las grandes epidemias de la vida laboral moderna ${ }^{1}$, derivada principalmente de las profundas transformaciones que el trabajo ha presentado en las últimas décadas, especialmente desde la década del ' $80^{2}$, caracterizadas por una fuerte intensificación, precarización e inestabilidad laboral, como estrategias impulsadas para otorgar mayor adaptación de las organización a partir de la productividad.

Dicho escenario, ha generado cambios en la esfera sociosimbólica de la relación persona- trabajo ${ }^{2}$, generando consecuencias a nivel físico y mental como respuesta a tales entornos, siendo el estrés un indicador de relevancia por su rol precursor en consecuencias organizacionales como accidentabilidad laboral ${ }^{3-5}$, ausentismo ${ }^{6}$, baja satisfacción en el trabajo $^{7}$, resistencia a los $\operatorname{cambios}^{8}$, acoso laboral ${ }^{9}$, entre otras.

La relación entre los aspectos psicosociales del trabajo y la salud laboral ha sido sólidamente documentada ${ }^{10-12}$, con indicadores físicos ${ }^{13-16}$ y psicológicos ${ }^{17-20}$. En consecuencia, resulta conveniente y necesaria su identificación, evaluación y control con el fin de evitar los riesgos asociados para la salud y la seguridad en el trabajo ${ }^{21,22}$.

Según la Organización Mundial para la Salud y la Organización Internacional del Trabajo $^{23}$, los riesgos psicosociales laborales son hechos, situaciones o contextos con una clara probabilidad de dañar la salud física, social o mental del trabajador de forma importante. En efecto, su característica fundamental se centra en la capacidad de ocasionar daño, en conjunto con la alta probabilidad de aparecer y de generar consecuencias graves para la salud. Esta conceptualización, es compartida por el Instituto Sindical de Trabajo, Ambiente y Salud de España ${ }^{24}$, el Instituto Nacional de Salud e Higiene en el Trabajo de España $^{25}$ y diversos autores ${ }^{26-28}$ consolidando al constructo como un fenómeno de relevancia organizacional, social y política.

Rodríguez ${ }^{29}$, plantea que constituyen factores de riesgos psicosociales laborales, los ritmos y exigencias de trabajo excesivas, urgencia temporal, trato inapropiado o injusto, inseguridad contractual, bajo control sobre el propio trabajo, exigencias contradictorias o bajo apoyo social, pues crean condiciones laborales disfuncionales para el individuo y la organización, la mayoría de las veces invisibilizadas, constantes y difíciles de pesquisar, lo que estaría en concordancia con las actuales condiciones laborales a las que están expuestos trabajadores de distintos sectores laborales en el país.

El estrés es una de las consecuencias de las condiciones adversas en el lugar de trabajo y esta relación ha sido documentada por diversas investigaciones ${ }^{30-32}$. El estrés percibido es una particular manifestación del estrés, el cual se caracteriza por el grado en que las situaciones de la vida se perciben como impredecibles e incontrolables, y por tanto se valoran como estresantes ${ }^{33}$. Este estrés agudo surge a partir de la evaluación cognitiva que realiza el sujeto del suceso como de los recursos propios para afrontar dicha situación, la que es considerada como amenazante (Vera y Wood, 1994; tesis de grado no publicada). En ese sentido, las condiciones organizacionales pudieran ser interpretadas como estresores en la medida que se les otorga una connotación negativa a la cual el trabajador no puede hacer frente.

Si bien, las investigaciones de las condiciones psicosociales laborales en el país, se encuentran en un estado incipiente, los estudios se han concentrado en el sector minero ${ }^{34-36,}$ en el sector del retail y supermercados ${ }^{37,38}$, en el área de la salud pública ${ }^{39,40}$ y en el colectivo de mujeres ${ }^{41,42}$ siendo la temática menos explorada en el sector energético.

En Chile, el abastecimiento del consumo energético es clave para el desarrollo económico y social del país ${ }^{43}$. En consecuencia, su avance anticipa crecimiento y empleabilidad $^{44}$, no obstante el sector presenta tasas de accidentabilidad del 3,5\% y mortalidad por accidentes del trabajo de $6,3 \%$ lo que supera al promedio nacional ${ }^{45}$, por consiguiente no resultan indiferente los aspectos y condiciones laborales en las que se 
desempeña los trabajadores ligados a esta industria, así como el posible efecto en su salud mental.

El presente estudio, analiza la relación entre los factores psicosociales de trabajo y el estrés percibido, a partir del instrumento Suseso Istas 21 perteneciente al Protocolo de Vigilancia de Riesgos Psicosociales del Trabajo ${ }^{22}$, el que se fundamenta en los Modelo Demanda-Control y Apoyo de Karasek \& Theorell ${ }^{46}$ y Desbalance Esfuerzo-Recompensa de Siegrist ${ }^{47}$, ambos modelos teóricos con amplia evidencia en materia de salud laboral.

\section{MATERIAL Y MÉTODOS}

\section{Diseño y procedimiento}

El estudio se basó en un diseño No Experimental, Selectivo «Ex post facto», de alcance descriptivo y correlacional. La técnica de muestreo empleada fue No probabilística por Conveniencia. La muestra estuvo constituida por 292 trabajadores de una empresa del sector energético, cuyo universo de trabajadores es 2227 , distribuida en las ciudades de Santiago, Iquique, Concepción y Valparaíso. De los cuales 241 son hombres $(82,5 \%)$ y 51 mujeres $(17,5 \%)$, con una edad promedio de 44,14 años (d.t.=11,12) concentrándose el 50\% entre los 40 y 60 años. A su vez, la antigüedad media fue de 16,79 (d.t.= 12,43). Respecto a la jornada laboral, la mayoría de los trabajadores realiza sus actividades en horario diurno $(81,8 \%)$ mientras que el $18,2 \%$ lo realiza en turnos rotativos (incluyendo el nocturno), siendo necesario destacar que sólo trabajadores hombres se encuentran bajo esta modalidad.

\section{Instrumentos}

- Cuestionario Suseso Istas 21. Versión Breve: Adaptado y validado para la población chilena por requerimiento de la Superintendencia de Seguridad Social de Chile -SUSESO- ${ }^{48}$. Mide 5 dimensiones correspondientes a: Exigencias Psicológicas, Trabajo Activo y Desarrollo de Habilidades, Apoyo Social y Liderazgo en la Empresa, Compensaciones y Doble Presencia, a través de 20 reactivos, con escala tipo Likert de 4 puntos, donde 0 representa "Nunca" y 4 "Siempre», correspondiendo una mayor puntuación a un mayor riesgo. El índice de fiabilidad del instrumento en este estudio fue de: $\alpha=0.769$.

- Escala de Estrés Percibido; adaptado y validado para la población chilena (Vera y Wood,1994). La escala abarca aspectos del estrés como: impredictibilidad, incontrolabilidad, sobrecarga y preguntas directas sobre niveles de estrés experimentados Cuenta con 14 ítems, con escala de respuesta tipo Likert de 4 puntos, donde 0 es "Nunca" y 4 es "Casi siempre». El índice de fiabilidad en este estudio fue de: $\alpha=0.843$.

\section{Variables de estudio}

Se integraron en el análisis variables sociodemográficas como edad, sexo y antigüedad laboral, así como las variables relativas a los factores psicosociales del trabajo.

- Exigencias Psicológicas: hacen referencia a aquellos aspectos cuantitativos y de tareas. El primero representado por el volumen del trabajo y el tiempo disponible para ejecutar las tareas, y el segundo representado por tareas cognitivas o emocionales ${ }^{48}$.

- Trabajo Activo y desarrollo de habilidades: alude a la autonomía, control de tiempos, y posibilidades de aplicar aprendizajes, en consecuencia refleja la influencia que el trabajador posee sobre su propio trabajo ${ }^{48}$.

- Apoyo social y calidad de liderazgo: refiere a las relaciones interpersonales y con superiores en el trabajo. El apoyo social representa el aspecto funcional y el sentimiento de grupo, el componente emocional ${ }^{48}$. 
- Compensaciones: se asocia con la percepción de seguridad y continuidad laboral, así como también con el nivel status, estima y el salario percibido ${ }^{48}$.

- Doble Presencia: alude al nivel de exposición a doble trabajo, asociado al ámbito laboral y doméstico-familiar ${ }^{48}$.

- Estrés percibido: Refiere a la connotación cognitiva y emocional frente un cambio o una situación en particular y su capacidad de afrontarla, considerando su impredictibilidad, incontrolabilidad y sobrecarga, como el nivel de estrés experimentado (Vera y Wood, 1994).

\section{Procedimiento}

Para la recolección de los datos, las aplicaciones fueron en grupos según áreas de trabajo. Se realizó un encuadre teórico respecto al contenido y objetivos del estudio y se proporcionó a cada participante un consentimiento informado junto a un cuadernillo con los dos instrumentos, con respuesta anónima y sólo indicación de variables sociodemográficas. El tiempo estimado para la ejecución del procedimiento fue de 45 minutos en el lugar de trabajo, supervisado por el investigador.

\section{Técnicas de análisis de los datos}

Se utilizó el paquete estadístico SPSS versión 17 y se desarrollaron análisis Univariado, pruebas de diferencias de medias para muestras no paramétricas y análisis de correlaciones. Se analizó la asociación entre las variables mediante la matriz de correlaciones de Spearman, por tratarse de variables ordinales y por contar con escalas distribuidas de forma distintas a la normal.

\section{RESULTADOS}

Respecto a los niveles de riesgos psicosociales presentes en la organización estudiada, es posible señalar, a nivel descriptivo, que la dimensión doble presencia es aquella en el nivel más alto de riesgo (44\%), sigue la dimensión apoyo social y compensaciones $(34,2 \%$ respectivamente) y las dimensiones exigencias psicológicas y trabajo activo presentan en un $32,2 \%$ y $31,5 \%$ respectivamente, un nivel alto de riesgo psicosocial (Figura 1).

Figura 1. Frecuencia de exposición a los riesgos psicosociales en trabajadores del sector energético

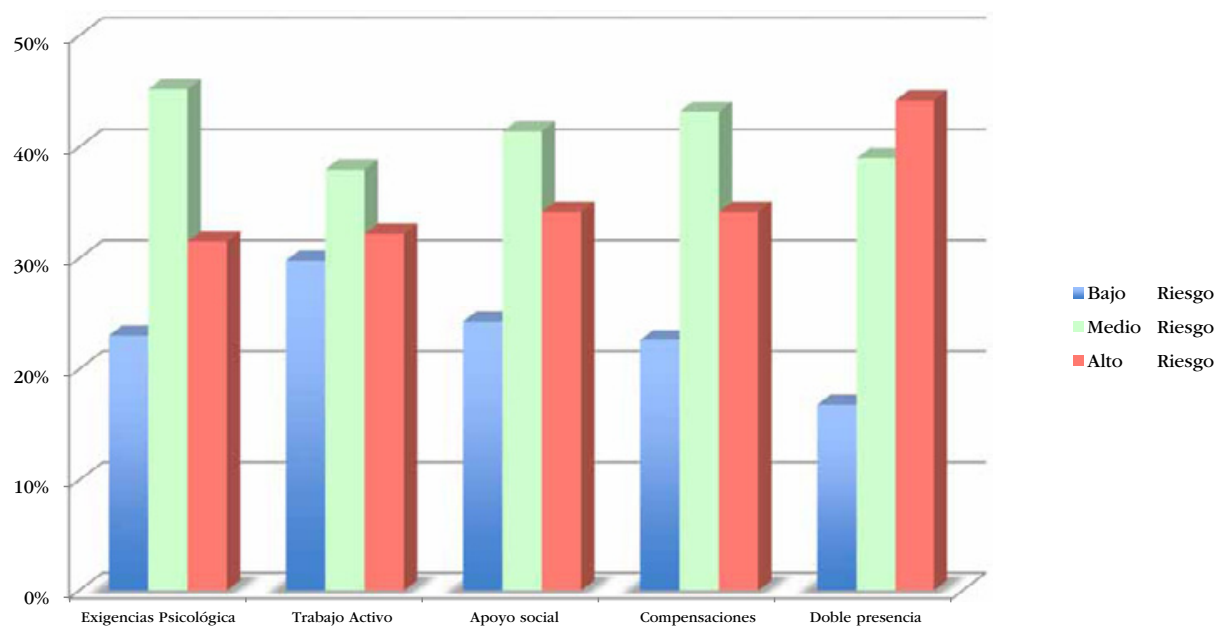

A nivel comparativo, la tabla I, presenta los resultados descriptivos y correlaciones de las variables estudiadas. De acuerdo a los estadísticos de tendencia central, es posible señalar que ninguno supera la media teórica de las escalas, por el contrario los factores de riesgos psicosociales del trabajo y el estrés autoinformados son relativamente bajos en esta muestra. Por lo tanto, es plausible hipotetizar al menos dos razones para estos 
resultados, la primera asociada al frecuente subreporte que presenta la temática y la segunda razón puede estar dada porque la empresa como parte de sus políticas organizacionales ha implementado distintos programas y/o beneficios ligados a la salud ocupacional, situación que podría incidir en percepciones menos críticas al sistema laboral.

Tabla I. Estadísticos descriptivos y matriz de correlaciones del estudio( $N=292$ )

\begin{tabular}{|c|c|c|c|c|c|c|c|c|}
\hline & M & DT & 1 & 2 & 3 & 4 & 5 & 6 \\
\hline 1. EPC & 19,76 & 6,98 & 1 & & & & & \\
\hline 2. $\mathrm{EP}$ & 10,27 & 2,43 &, $356(* *)$ & 1 & & & & \\
\hline 3. TA & 7,1 & 2,83 &, $173^{(* *)}$ & ,102 & 1 & & & \\
\hline 4. AS & 5,78 & 2,87 &, 360 (**) &, $\left.256^{* * *}\right)$ &, $415(* *)$ & 1 & & \\
\hline 5. $\mathrm{CO}$ & 4,46 & 2,29 & ,299(**) &, $214(* *)$ &, $207(* *)$ &, $284(* *)$ & 1 & \\
\hline 6. DP & 3,23 & 1,73 &, 197 (**) $^{* *}$ & 098 &, $122\left(^{*}\right)$ & 0,093 &, $133\left(^{*}\right)$ & 1 \\
\hline
\end{tabular}

Fuente: Elaboración propia

Nota: EPC: Estrés Percibido; EP: Exigencias Psicológicas; TA: Trabajo Activo; AS:

Apoyo Social; C: Compensaciones; DP: Doble Presencia.

$(\mathrm{n}=292 ; * * \mathrm{p}<.01 ; * \mathrm{p}<.05)$

La matriz de correlaciones (Tabla I), revela que todos los factores psicosociales del trabajo estudiados presentan asociaciones positivas y significativas con estrés percibido, siendo las dimensiones de mayor asociación el apoyo social y exigencias psicológicas (rho =,360 $p<0.01$; rho =,356 $p<0.01$ ), compensaciones (rho =,299 $p<0.01$;) y las de menor asociación son trabajo activo y doble presencia (rho =,173 $p<0.01$; rho $=0,197$ $p<0.01$ respectivamente). Por otra parte, la mayoría de las dimensiones psicosociales del trabajo presentan asociaciones significativas entre si, excepto las dimensiones de trabajo activo y doble presencia con exigencias psicológicas, así como también doble presencia y apoyo social, entre quienes se observan asociaciones reducidas y no significativas.

Por otra parte, también se ha intentado identificar la posible existencia de asociaciones significativas entre las características sociodemográficas y las variables estudiadas (Tabla II) encontrando a través de la prueba U de Mann Whitney, que sólo la dimensión doble presencia presenta diferencias significativas según sexo $(U=4989,500 ; Z=-2,289$, $p<0,022)$ presentando el grupo de mujeres mayor exigencias en esta dimensión. La misma prueba estadística evidencia diferencias estadísticamente significativas de la dimensión compensaciones y sistema de turno ( $U=5064,500 ; Z=-2,449, p<0,014)$ siendo mayor estos factores de riesgo en el grupo de aquellos trabajadores con este tipo de modalidad laboral. Igualmente, a través del mismo procedimiento, se constató que existen diferencias significativas entre las dimensiones de trabajo activo, doble presencia y antigüedad laboral ( $U=8730,5 ; Z=-2,044, p<0,04 ; U=7762 ; Z=-3,584, p<0,0003$ respectivamente), siendo aquellos con menor antigüedad ( 9 años) quienes presentan mayores dificultades con dimensión trabajo activo y doble presencia.

Por otra parte, la prueba Kruskall Wallis, muestra diferencias significativas entre las dimensiones de compensaciones y doble presencia según edad $\left(X^{2}(3)=15,134\right), p<.002$; $\left(X^{2}(3)=17,042\right), p<.001$ respectivamente). A través de la Corrección por Bonferroni $(p=0,05 / 4)$ y la prueba estadística U de Mann Whitney, aplicada a los cuatro grupos etarios por proceso de pares, se logró establecer que las diferencias estadísticamente significativas en cuanto a la dimensión compensación se encuentran entre las edades de adulto joven y adulto ( $U=773,500, Z=-3,040, p=0,002)$, esto es, el grupo de menor edad presenta mayores riesgos en la dimensión compensación que el grupo adulto ( $M=80,06>57,39$ respectivamente). Otros grupos que presentaron diferencias significativas fue la categoría adulto y adulto medio $(U=5562,500, Z=-3,240, p=0,001)$, siendo la categoría adulto medio la que presenta la media mas alta, esto es, mayor riesgo en compensaciones $(M=134,16>106,26)$. El mismo procedimiento se aplicó para 
identificar diferencias de medias de la dimensión doble presencia en los cuatro grupos etarios, encontrándose diferencias significativas sólo para los casos de adulto joven y adulto tardío $(U=136,500, Z=-3,203, p=0,001)$, adulto y adulto medio ( $\mathrm{U}=5926,000$, $Z=-2,552, p=0,011)$ y adulto- adulto tardío $(U=625,500, Z=-3,351, p=0,001)$. En el primer caso la diferencia de media es mas alta para la categoría adulto joven $(M=29,54>17,70)$, en el segundo caso la mayor media la presenta la categoría adulto medio $(M=136,03>114,31)$ y en el tercer caso la diferencia de media es mas alta en el grupo adulto $(M=65,12>39,93)$.

Tabla II. Pruebas Estadísticas no paramétricas

\begin{tabular}{|c|c|c|c|c|c|c|}
\hline & & EP & TA & AS & C & DP \\
\hline \multirow[t]{4}{*}{ Sexo } & U de Mann-Whitney & 5489,5 & 6033,5 & 5763 & 5211,5 & 4989,5 \\
\hline & Z & $-1,288$ & $-0,217$ & $-0,747$ & $-1,83$ & $-2,289$ \\
\hline & Sig. asintót. (bilateral) & 0,198 & 0,828 & 0,455 & 0,067 & 0,022 \\
\hline & & EP & TA & AS & C & DP \\
\hline \multirow[t]{4}{*}{ Sistema de turno } & U de Mann-Whitney & 5965,5 & 6006 & 6181 & 5064,5 & 6273 \\
\hline & Z & $-0,712$ & $-0,626$ & $-0,293$ & $-2,449$ & $-0,118$ \\
\hline & Sig. asintót. (bilateral) & 0,476 & 0,531 & 0,769 & 0,014 & 0,906 \\
\hline & & EP & TA & AS & C & DP \\
\hline \multirow[t]{3}{*}{ Tramos etarios } & Chi-cuadrado & 1,082 & 4,719 & 0,572 & 15,134 & 17,042 \\
\hline & $\mathrm{gl}$ & 3 & 3 & 3 & 3 & 3 \\
\hline & Sig. asintót. & 0,781 & 0,194 & 0,903 & 0,002 & 0,001 \\
\hline
\end{tabular}

a Prueba de Kruskal-Wallis

\begin{tabular}{lllllll}
\hline & & EP & \multicolumn{1}{c}{ TA } & \multicolumn{1}{c}{ AS } & \multicolumn{1}{c}{ C } & \multicolumn{1}{c}{ DP } \\
\hline Antigüedad & Chi-cuadrado & 1,691 & 7,841 & 0,172 & 2,834 & 14 \\
& gl & 2 & 2 & 2 & 2 & 2 \\
& Sig. asintót. & 0,429 & 0,02 & 0,917 & 0,242 & 0,001 \\
& a Prueba de Kruskal-Wallis & & & & & \\
\hline
\end{tabular}

Fuente: Elaboración propia

Nota: EPC: Estrés Percibido; EP: Exigencias Psicológicas; TA: Trabajo Activo; AS: Apoyo Social; C: Compensaciones; DP: Doble Presencia.

\section{DISCUSIÓN}

A la luz de los resultados, es posible señalar que el estrés percibido presenta relaciones significativas con todos los factores de riesgos psicosociales del trabajo estudiados, lo que es coherente con estudios previos en materia de estrés ${ }^{30-32}$. Destacan la mayor magnitud de las asociaciones de apoyo social y las exigencias psicológicas con estrés percibido, esto es, a medida que se vuelve mas crítica la percepción del apoyo de pares o del líder, más aumenta el estrés percibido en este grupo de trabajadores. Del mismo modo, mientras mayor es la percepción de desequilibrio entre volumen de trabajo y tiempo disponible para su ejecución, así como mayores sean las exigencias cognitivas y emocionales, también será mayor el estrés percibido en este grupo. No obstante, esta situación no sorprende, ya que en empresas del rubro energético buena parte del trabajo requiere una coordinación fluida entre los sistemas, por lo tanto, la comunicación y el sentimiento de apoyo de pares resultan fundamentales para este tipo de trabajo. Lo mismo ocurre con el factor exigencias psicológicas las que naturalmente presentan una 
alta asociación con estrés percibido, debido a la alta carga cognitiva que demanda un trabajo cruzado por el uso sistemático de tecnologías.

Por otra parte, es posible señalar que las variables sociodemográficas presentan un rol significativo con algunos factores de riesgos psicosociales del trabajo estudiados. Destacando el caso de las mujeres quienes presentan una mayor magnitud del factor doble presencia. Esto es coincidente con estudios previos, donde la interferencia del trabajo sobre la vida privada-familiar, es particularmente exigente con ellas ${ }^{49}$. Lo que está en directa relación con el mayor número de horas que aquellas asignan a las labores domésticas, culturalmente asociadas al rol de género ${ }^{50}$. Esta evidencia reviste importantes consecuencias para la salud mental de estas mujeres trabajadoras, emergiendo como un colectivo con mayor vulnerabilidad, por cuanto, medidas organizacionales que se orienten al equilibrio saludable en materia de vinculación trabajo-vida privada y familia se constituye como un reto necesario para una gestión moderna.

Así mismo, la antigüedad laboral, presenta una asociación ya descrita en estudios previos con trabajo activo y doble presencia ${ }^{49}$, es decir, los trabajadores con menor antigüedad perciben problemas de autonomía, escasas posibilidades de desarrollo, así como también mayor interferencia del trabajo con su vida privada. Esto no es fortuito, ya que se trata de un colectivo con menor experiencia laboral, lo que justifica la presencia de mayor control de parte de las organizaciones. Igualmente, las aspiraciones de los trabajadores más jóvenes coexisten con sus expectativas de desarrollo profesional, lo que es propio de la etapa de trabajo en la cual se encuentran. Del mismo modo, esta situación coincide con el proyecto familiar y/o independencia del grupo de menor antigüedad, lo que naturalmente se asocia con dificultad para conciliar ambas áreas en este grupo de trabajadores.

En cuanto a la edad, los análisis indican que tanto las compensaciones como la doble presencia son percibidas de forma significativamente distintas según la edad de los trabajadores, siendo en general la percepción más crítica en los grupos menos envejecidos, lo que está muy relacionado con las razones esgrimidas anteriormente.

Por otra parte, aquellos trabajadores que ejecutan sus labores con sistema de turno tienen una visión más crítica de las compensaciones laborales, que aquellos que no trabajan bajo estas condiciones. Esto resulta consistente con la literatura, puesto que la falta de reconocimiento formal e informal en conjunto con la inestabilidad, inseguridad laboral o trabajos temporales, actúan como un estresor de relevancia para la salud psicológica de los trabajadores, incluso con incidencia en enfermedades coronarias ${ }^{51}$, afecto positivo ${ }^{52}$, así como es fuente de resultados negativos individuales $y$ organizacionales $^{53}$. Por lo tanto, este colectivo de trabajadores estaría expuesto en mayor medida a resultados negativos tanto individuales como organizacionales, sólo por desarrollar sus labores en turnos rotativos o nocturnos, lo cual reviste particular importancia por tratarse de un sector cuyos niveles de crecimiento y empleabilidad van en alza, de manera que sus niveles de exposición estarían en la misma dirección.

Por último, los análisis de frecuencia revelan que existe riesgo elevado de estrés psicosocial en las cinco dimensiones evaluadas, en efecto, al menos 3 de cada 10 trabajadores presentan alto riesgo en alguno de los factores psicosociales. Siendo el factor doble presencia el que presenta mayor grado. En consecuencia, dada la magnitud de los niveles de alto riesgo en exigencias psicológicas, trabajo activo y desarrollo de habilidades, apoyo social y calidad de liderazgo, compensaciones y doble presencia, esta muestra de trabajadores pueden presentar daño tanto en su salud física como psicológica. Por consiguiente, resulta relevante un trabajo de intervención a nivel organizacional, que considere cambios en aspectos del trabajo como la carga laboral y la definición de plazos razonables de entrega de tareas, posibilidades de mayor autonomía en el trabajo de los grupos etarios mas jóvenes, programas de mayor flexibilidad de horarios para el grupo de mujeres, entre otras, de modo de evitar consecuencias organizacionales y de salud en estos grupos ocupacionales. 
Cabe señalar, que si bien los resultados parecen estar en la misma línea de otros estudios similares en la materia, no es posible llegar a conclusiones más categóricas, por la naturaleza de los datos. Se sugiere por lo tanto, continuar con la exploración de tales variables en sectores económicos menos explorados, así como en particular prestar atención en las consecuencias de los actuales entornos laborales sobre el colectivo de mujeres trabajadoras, joven y adulto joven y aquellos colectivos que desarrollan labores en sistema de turnos, por tratarse de grupos que consistentemente presentan mayor exposición a riesgos psicosociales laborales y por ende resultados mas adversos de salud. De igual modo, es relevante señalar que el estudio se realizó sobre una población de trabajadores específicos y basado en autoreporte considerando la respuesta de sólo quienes están laboralmente activos y con un estado de salud físico y emocional que les permite desempeñarse a diario, omitiendo la percepción de aquellos trabajadores ausentes. Es por ello que es necesario en futuros estudios incluir indicadores de carácter objetivo, tales como: licencias médicas, tasas de rotación, sobre tiempo, accidentabilidad laboral de la organización, entre otros, de manera de complementar los estudios en la materia, así como medio para advertir la subnotificación.

Por otro lado, también es importante resaltar que la muestra se caracterizó por ser no probabilística, aspecto que pudiera haber influenciado en que las variables de estudio no se distribuyeran normalmente y con ello variar en la interpretación de los indicadores.

Finalmente, señalar que si bien este tipo de evaluaciones pueden suscitar resistencias iniciales para las organizaciones, las mismas contribuyen a resultados organizacionales y personales en un contexto de sustentabilidad, particularmente si se integran sobre la base de políticas que promuevan la relación de equilibrio entre persona y trabajo.

\section{REFERENCIAS BIBLIOGRÁFICAS}

1. Houtman, Jettwghoff y Cedillo. Sensibilizando sobre el Estrés Laboral en los Países en Desarrollo. Protección de la Salud de los Trabajadores Serie No. 6. WHO 2008.

2. Stecher, A. El campo de la investigación sobre transformaciones del trabajo, identidades y subjetividad en la modernidad contemporánea. Apuntes desde Chile y América Latina. En Stecher, A. \& Godoy, L. "Transformaciones del trabajo, subjetividad e identidades». Ril editores: Santiago, Chile; 2014.

3. García- Layunta, M., Oliver, A., Tomás, J., Verdú, F. \& Zaragoza, G. Factores Psicosociales Influyentes en la Ocurrencia de Accidentes Laborales. Arch. Prev. Riesgos Labor. 2002; 5(1):4-10.

4. Gil-Monte, P. Algunas Razones para considerar los Riesgos Psicosociales en el Trabajo y sus consecuencias en la Salud Pública. Rev. Esp. Salud Pública 2009; 83: 169-173.

5. Rentería J., Fernández, E., Tenjo, A. \& Uribe, A. Identificación de factores psicosociales de riesgo en una empresa de producción. Rev Diversitas 2009; 5 (1): 161- 175.

6. Gimeno, D., Marko, D., \& Martínez, J. Relación entre los factores de riesgo psicosociales laborales y la ausencia por razones de salud: motivos laborales y no laborales en España. Archivo de Prevención de Riesgos Laborales 2003; 6: 139- 145.

7. Martín-García, J., Luceño, L., Jaén, M. \& Rubio, S. Relación entre factores psicosociales adversos, evaluados a través del cuestionario multidimensional Decore, y salud laboral deficiente. Rev. Psicothema. 2007; 19: 95- 101.

8. García, M., Rubio, P. \& Bravo, L. Relación entre los factores de riesgo psicosocial y la resistencia al cambio organizacional en personal de seguridad de una empresa del sector económico terciario. Rev. Diversitas 2007; 3: 301- 315.

9. Segurado, A., Agulló, E., Rodríguez, J., Agulló, M., Boada, J. \& Medina, R., Las Relaciones Interpersonales como Fuente de Riesgo de Acoso Laboral en la Policía Local. Rev. Psicothema. 2008; 20: 739- 744.

10. OSHA. European Agency for Safety and Health at Work. EUROPEAN RISK OBSERVATORY REPORT. European Survey of Enterprises on New and Emerging Risk; 2010 osha.europa.eu/en/publications/ reports/esener1_osh_management [ Links ]

11. Houdmont, J., y Leka, S. Occupational Health Psychology.1: John Wiley \& Sons; 2010.

12. Näswall, K., Hellgren, J., \& Sverke, M. The individual in the changing working life. Cambridge: Cambridge University Press; 2008. 
13. Juárez-García, A. Factores Psicosociales Laborales relacionados con la Tensión Arterial y Síntomas Cardiovasculares en Personal de Enfermería en México. Rev. Salud Públ. Méx. 2007; 49:109-117.

14. Marreno, M. Estrés psicosocial como factor de riesgo para el peso al nacer en trabajadoras embarazadas en la edad juvenil. Rev. Cubana de Salud y Trabajo 2007; 8: 20-26.

15. Escribá- Agüir, V. Ambiente Psicosocial y Salud de los Trabajadores. Arch Prev Riesgos Labor 2005; 9: 6-9.

16. Aranda, C., Pando, M., Torres, T., Salazar, J. \& Sánchez, J. Factores Psicosociales y Patologías Laborales en Trabajadores de un Sistema de Transporte Público Urbano, México. Rev. Col. Psiqui. 2011; 40: $266-278$.

17. Guic, E., Mora, O., Rey, R., \& Robles, A. Estrés Organizacional y Salud en funcionarios de Centros de Atención Primaria. Rev. Med. Chile 2006; 134: 447-455.

18. Gómez, V. Factores Psicosociales del Trabajo y su relación con la Salud Percibida y la Tensión Arterial: Un estudio con maestros escolares en Bogotá, Colombia. Rev Cienc. Trab 2008; 30: 132- 137.

19. Leka \& Jain. Health Impact of Psychosocial Hazards at Work: An Overview. WHO; 2010.

20. Ansoleaga, E., Sintomatología depresiva y consumo riesgoso de alcohol en trabajadores expuestos a factores psicosociales laborales adversos: Un asunto pendiente en Salud Pública. Tesis para optar al grado de Doctor, Escuela Salud Pública, Universidad de Chile, Santiago, Chile; 2013.

21. OSHA European Agency for Safety and Health at Work. European Risk Observatory Report. Expert Forecast on emerging Psychosocial risks related to occupational safety and health; 2007. osha.europa.eu/ en/publications/reports/7807118. [ Links ]

22. Ministerio de Salud. Protocolo de Vigilancia de Riesgos Psicosociales en el Trabajo MINSAL. 2013 [Consultado 23 de Junio de 2014]; Disponible en: http://web.minsal.cl/portal/url/item/e03977235675788 $6 \mathrm{e} 040010165014 a 72 . p d f$

23. OIT-OMS. Factores psicosociales en el trabajo: Naturaleza, Incidencia y Prevención. Informe del Comité Mixto OIT-OMS sobre Medicina del Trabajo. Novena reunión Ginebra, 18-24 de Septiembre de 1984

24. Instituto Sindical de Trabajo Ambiente y Salud, ISTAS. Manual de la versión media del método ISTAS-21 (CoPsoQ) de evaluación de riesgos psicosociales; 2002

25. INSHT. Instituto Nacional de Salud e Higiene en el Trabajo de España. VI Encuesta nacional de Condiciones de trabajo. Madrid: Ministerio de Trabajo y Asuntos Sociales. 2010.

26. Acevedo G, Farías A, Sánchez J. Trabajar en el Sector Salud: Factores influyentes en sus condiciones y medioambiente de trabajo. Facultad de Filosofía y Humanidades de la Universidad Nacional de Córdoba; 2011.

27. Gil Monte. Riesgos psicosociales en el trabajo y salud ocupacional. Rev. Perú Med. Exp. Salud Pública 2012; 29(2): 237-241.

28. Meliá JL, Nogareda C, Lahera M, Duro A, Peiró JM, Pou R, Salanova, Gracia D, de Bona JM, Bajo JC, Martínez-Losa F. Principios comunes para la evaluación de riesgos psicosociales en la empresa. Perspectivas de Intervención en Riesgos Psicosociales; Evaluación de Riesgos. Barcelona: Foment del Treball Nacional; 2006. 13-36.

29. Rodríguez, M. Factores Psicosociales de Riesgo Laboral: ¿Nuevos Tiempos, Nuevos Riesgos? Observatorio Laboral Rev. Venezolana 2009; 2: 127- 141.

30. Fischer R, Boer D. What is more important for national well-being: money or autonomy? A meta-analysis of well-being, burnout, and anxiety across 63 societies. J. Pers. Soc. Psychol. 2011; 101(1):164-184.

31. Salanova M, Grau R, Martínez I. Demandas Laborales y Conductas de Afrontamiento: El Rol Modulador de la Autoeficacia Profesional. Rev. Psicotbema 2005; 17: 390-395.

32. Organización Internacional del Trabajo OIT. Riesgos emergentes y nuevos modelos de prevención. Ginebra; 2010

33. Remor, E. Psychometric properties of a European Spanish version of the Perceived Stress Scale (PSS). Span.J. Psychol. 2006; 9(1): 86-93.

34. Gómez P., Hernández J., Méndez M. Factores de Riesgo Psicosocial y Satisfacción Laboral en una Empresa Chilena del Área de la Minería. Rev. Cienc Trab. 2014; 16 (49):9-16.

35. Ansoleaga E. Factores psicosociales laborales asociados a riesgo de sintomatología depresiva en trabajadores de una empresa minera. Rev. Salud de los Trabajadores 2010; 18: 7-16.

36. Vera A, Sepúlveda R, Contreras G. Autorreporte de síntomas físicos y correlatos psicosociales en trabajadores de la minería. Cienc Trab. 2006; 8: 74-78.

37. Stecher, A. La Modernización de la Industria del Retail: Reorganización empresarial y experiencias laborales. En J. Ossandón \& E. Tironi (Edits.) Adaptación. La empresa chilena post-Friedman. Santiago de Chile: UDP; 2013. 
38. Stecher, A; Godoy, L.; \& Toro, J. P. Sociabilidad y calidad de vida laboral en un entorno de trabajo flexible. La experiencia de trabajadores de supermercado en Santiago de Chile. Rev. Psicología Univ. Chile. 2012; 21(2):133-157

39. Godoy, L., Stecher, A., Toro, J. P. \& Ansoleaga, E., Condiciones de trabajo, estilos de gestión y bienestar psicosocial: la percepción de médicos y enfermeras de hospitales públicos en Santiago de Chile. En E. Ansoleaga; O. Artaza; J. M. Suárez (Eds). Personas que cuidan personas: Dimensión bumana y trabajo en salud. Santiago de Chile: OPS/OMS; 2012. 183-196

40. Durán, G. Doniez, V. Páez, A. Zanga, R. Stecher, A. Godoy, L. Toro, JP. Artaza, O. \& Ansoleaga, E. Breve caracterización de trabajadoras y trabajadores de la salud en Chile. Personas que cuidan personas: Dimensión humana y trabajo en salud. Santiago de Chile: OPS/OMS; 2012:102-106

41. Díaz X. y Mauro A. En E. Ansoleaga; O. Artaza; J. M. Suárez (Eds.) Riesgos psicosociales y salud mental de trabajadores y trabajadoras del sector salud en Chile. Personas que cuidan personas: Dimensión humana y trabajo en salud. Santiago de Chile: OPS/OMS; 2012: 146-164

42. Elisa Ansoleaga y Lorena Godoy. La maternidad y el trabajo en Chile: Discursos actuales de actores sociales. Rev. Polis 2013; 12 (35): 337-356 [consultado 15 de octubre de 2014; Disponible en: http://polis. revues.org/9102\#article-9102

43. CNE. Comisión Nacional de Energía. 2010 [Consultado 20 de Mayo de 2014]; Disponible en: http://www. cne.cl/noticias/otros/301-ministro-raineri-forma-comite-de-seguridad-energetica.

44. Ministerio de Energía de Chile. Estrategia Nacional de Energía 2012-2030. 2012 [Consultado 9 de Abril de 2014]; Disponible en: http://www.minenergia.cl/estrategia-nacional-de-energia-2012.html.

45. SUSESO. Registro de Accidentes Laborales Fatales (RALF) y Boletines estadísticos. Estadísticas de Accidentabilidad [En línea]. 2013 [Consultado 8 de Mayo 2014]. Disponible en: http://www.suseso.cl/ Repositorio/20839\%5C2014_04_28_Estad\%EDsticas\%20de\%20Accidentabilidad\%202013.pdf

46. Karasek, R. \& Theorell, T. Healthy Work: Stress, Productivity, and the Reconstruction of Working Life. New York: Basic books; 1990.

47. Siegrist, J. Adverse Health Effects of High- Effort/ Low-Reward Conditions. J Occup Health Psychol.1996; 1: $24-41$.

48. Alvarado R, Pérez-Franco JM, Saavedra N, Fuentealba C, Alarcón A, Marchetti N. y Aranda W. Validación de un cuestionario para evaluar riesgos psicosociales en el ambiente laboral en Chile. Rev. Méd. Chile 2012; 140: 1154- 1163

49. Acevedo G., Sánchez J., Farías M, Fernández A. Riesgos Psicosociales en el Equipo de Salud de Hospitales Públicos de la Provincia de Córdoba, Argentina. Cienc Trab 2013; 15(48):140-147

50. Carrasquer, P. La doble presencia. El trabajo y el empleo femenino en las sociedades contemporáneas. Tesis Doctoral, Departamento de Sociología, Universidad Autónoma de Barcelona; 2009.

51. Virtanen M, Nyberg S, Batty PD, Jokela M, Heikkila K, Fransson E, et al. Perceived job insecurity as a risk factor for incident coronary beart disease: systematic review and meta-analysis BMJ [en linea]. 2013.347: f4746. [Consultado 11 de Sep de 2014.

52. Vander, E. T., Bosman, J., De Cuyper, N., \& De Witte, H. Does Positive Affect Buffer the Associations between Job Insecurity and Work Engagement and Psychological Distress? A Test among South African Workers. Appl. Psychol. Int Rev. 2013; 62:558-570.

53. De Cuyper, N., De Jong, J., De Witte, H., Isaksson, K., Rigotti, T., \& Schalk, R. Literature review of theory and research on the psychological impact of temporary employment: Towards a conceptual model. Int. J. Manag. Rev. 2008; 10: 25-51. 